\title{
An Inventory Model for Constant Demand with Shortages under Permissible Delay in Payments
}

\author{
R.Amutha ${ }^{1}$, Dr.E.Chandrasekaran ${ }^{2}$ \\ ${ }^{1}$ (Research Scholar, Department of Mathematics, presidency college-05) \\ ${ }^{2}$ (Associate Professor, Department of Mathematics, presidency college-05)
}

\begin{abstract}
This paper presents an inventory model for deteriorating products with constant demand and time varying deteriorating rate, under permissible delay in payments. It discussed in two cases whether the permissible periods are less than or equal to or greater than replenishment cycle. During the permissible period both supplier and retailer got some benefits. Shortages are allowed and are completely backlogged. This model is explained with numerical example and sensitivity analysis.
\end{abstract}

Keywords - Deteriorating Products, Economic Order Quantity, Holding Cost, Permissible delay in periods.

\section{Introduction}

In Recent periods, researchers are developing inventory models for deteriorating items by introducing new concepts. For example there are few inventory models in association with salvage Value or disposal cost or sales revenue etc. Similarly researchers have developed trade credit with new ideas. This trade credit means that the supplier offers the customer (Case I) a permissible delay in payments to attract new customers to increase sales (Case II) and cash discount to motivate quick payment and reduce credit expenses. Case (I) has been derived under two different situations, (a) the permissible delay period less than or equal to replenishment cycle period for setting the account and (b) the permissible delay period is greater than replenishment cycle period for settling the account. Ouyang L-Y, C-T Chang and J-T Teng [14] developed an economic order quantity model under trade Credit .Mukesh Kumar, Anand Chauhan, Rajat Kumar [12] extended Ajantha Roy [1]model with trade credit for the purpose of maximizing the profit. Chaitanya Kumar Tripathy and Umakanta Mishra [2] and Shital S.Patel and R.D.Patel [17] gave inventory models with permissible delay in periods which include purchase cost and selling price with shortages and without shortages respectively. Kun -Shan Wu [8] made an attempt in his paper to obtain the optimal ordering quantity of deteriorating items for two parameters of Weibull distribution deterioration with shortages under permissible delay in payments. Manish pal and Sanjay Jain, Mukesh Kumar and Priya Advani [16], Tripathi R.P [20] discussed permissible delay in payments without deterioration. It means that they obtain an optimal ordering policy only for demand rate and not for any deterioration rate. Nita H.Shah, Poonam Pandey [13] prepared inventory model under delay in payment is permissible. Tripathy P.K, S.Pradhan [22] and Hesam Saiedy, Mohammed Bamani Moghadam [4] discussed partial backlogging in trade credit. Hardik Soni, Nita H.Shan and Chandra K.Jaggi [3] presented a review about trade credit. Trailokyanath Singh, Hadibandhu Pattanayak [19] formulated a model for exponentially declining demand under permissible delay in payments without shortages. Ravi Gor, Nita H Shah [15] developed a selling price dependent demand rate in which selling price is exponentially increasing with respect to time.

In our proposed model an attempt is made to develop an EOQ model for deteriorating items under permissible delay in payment with constant demand and time proportional deterioration rate. Shortages are allowed and are completely backlogged. Mathematical model has been developed in two cases. (I) The Credit period is less than or equal to the cycle time for settling the account and (ii) the credit period is greater than the cycle time for settling the account. Our aim is to minimize the total cost. Numerical examples are given and sensitivity analysis is carried out for changes in.

\section{ASSUMPTIONS AND NOTATIONS}

- The inventory system involves only one item

- $\quad$ Lead time is zero

- Shortages are not allowed

- Replenishment is instantaneous

- Delay in payment is allowed.

- Time horizon is infinite

- During time $T_{1}$ the inventory level becomes Zero due to demand and deterioration. At time $T_{1}$ the shortages start occurring.

- The demand for the item is constant with time. 
- $A=$ set up cost

- $Q=$ the initial order quantity

- $\quad h=$ holding cost per unit

- $\theta=\theta t$, time proportional deterioration rate.

- $C=$ the unit purchasing cost

- $I_{e}=$ The Interest earned per unit time

- $I_{c}=$ the interest Charged per unit time.

- $I(t)=$ Inventory level at any instant of time, $0 \leq t \leq T$

- $\quad T=$ Time interval (in Years) between two successive orders.

- $\quad M=$ Permissible period of delay in selling the accounts with the supplier.

- $C_{3}=$ The Inventory shortage cost per unit time

\section{MATHEMATICAL MODEL}

THE DIFFERENTIAL EQUATION THAT GOVERNS THE VARIATION OF INVENTORY WITH RESPECT TO TIME IS $\mathrm{I}(\mathrm{t})$

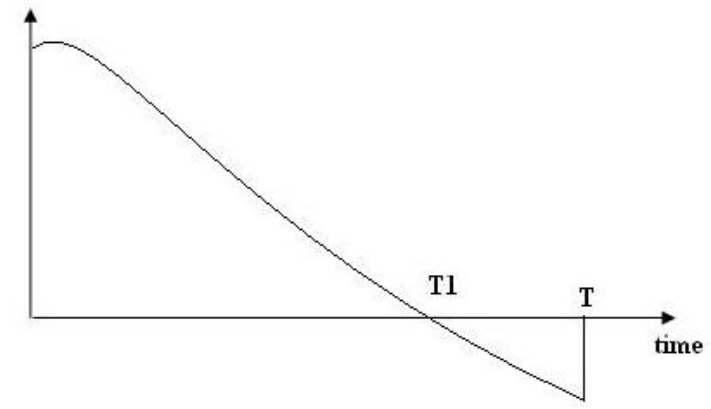

Fig 1

$$
\begin{aligned}
& \frac{d I(t)}{d t}+\theta t I(t)=-\mathrm{a}, 0 \leq t \leq T_{1} \\
& \frac{d I(t)}{d t}=-\mathrm{a}, \mathrm{T}_{1} \leq t \leq T
\end{aligned}
$$

With boundary condition $I\left(T_{1}\right)=0$ and $I(0)=Q$.

Solving equation (1)

I (t) $=\mathrm{a}\left(T_{1}-t\right)+\mathrm{a} \theta\left[\frac{T_{1}^{3}}{6}-\frac{t^{3}}{6}-\frac{T_{1} t^{2}}{2}+\frac{t^{3}}{2}\right]+\mathrm{a} \theta^{2}\left[\frac{T_{1}^{5}}{40}-\frac{t^{5}}{40}-\frac{T_{1}^{5} t^{2}}{6}+\frac{t^{5}}{6}+\frac{T_{1} t^{4}}{8}-\frac{t^{5}}{8}\right]$

Solving equation (2)

$\mathrm{I}(\mathrm{t})=\mathrm{a}\left(\mathrm{T}_{1}-\mathrm{t}\right)$

Shortage cost

$$
\begin{aligned}
\mathrm{SC} & =\int_{T_{1}}^{T} I(t) d t \\
& =-C_{3} \int_{T_{1}}^{T} a\left(T_{1}-t\right) d t \\
& =\frac{-a C_{3}}{2}\left[2 \mathrm{TT}_{1}-\mathrm{T}^{2}-\mathrm{T}_{1}{ }^{2}\right]
\end{aligned}
$$

Put $\mathrm{I}(0)=\mathrm{Q}$ in $(3)$

$\mathrm{Q}=\mathrm{aT}_{1}+\frac{a \theta T_{1}^{3}}{6}+\frac{a \theta^{2} T_{1} 5}{40}$

Deterioration cost

$\mathrm{DC}=\mathrm{C}\left(\mathrm{Q}-\int_{0}^{T_{1}} D(t) d t\right)$ 
$=-\mathrm{C}\left(\mathrm{Q}-\int_{0}^{T_{1}} a d t\right)$

$\mathrm{DC}=\mathrm{C}\left(\frac{a \theta T_{1}^{3}}{6}+\frac{a \theta^{2} T_{1} 5}{40}\right)$

Inventory holding cost

$\mathrm{HC}=\mathrm{h} \int_{0}^{T_{1}} I(t) d t$

$=\mathrm{h} \int_{0}^{T_{1}}\left[a\left(T_{1}-t\right)+a \theta\left(\frac{T_{1}^{3}}{6}-\frac{t^{3}}{6}-\frac{T_{1} t^{2}}{2}+\frac{t^{3}}{2}\right)+\mathrm{a} \theta^{2}\left(\frac{T_{1}^{5}}{40}-\frac{t^{5}}{40}-\frac{T_{1}^{3} t^{2}}{60}+\frac{t^{5}}{6}+\frac{T_{1} t^{4}}{8}-\frac{t^{5}}{40}\right)\right] \mathrm{dt}$

$=\frac{h}{T}\left[\frac{a T_{1}^{2}}{2}+\frac{a \theta T_{1}^{4}}{12}+\frac{11 a \theta^{2} T_{1}^{6}}{720}\right]$

The setup cost per cycle $: \frac{A}{T}$

Case I: $\mathrm{M} \leq \mathrm{T}_{1}$ (Payment at or before total depletion of inventory)

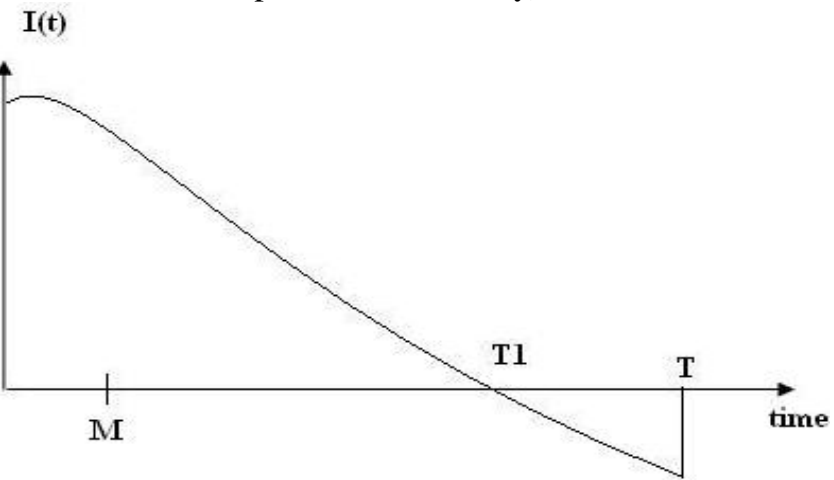

Fig. 2

In this case, the credit time expires on or before the inventory depleted completely to zero. The interest payable per cycle for the inventory not being sold after the due date $M$ is Interest payable in the horizon when $M<t \leq T_{1}$

$$
\begin{aligned}
\mathrm{IE}_{1} & =\mathrm{CI}_{\mathrm{e}} \int_{0}^{T_{1}}\left(T_{1}-t\right) d t \\
& =\frac{a C I_{e} T_{1}^{2}}{2} \\
\mathrm{IC}_{1} & =\mathrm{CI}_{\mathrm{c}} \int_{M}^{T_{1}} a d t \\
& =\mathrm{CI}_{\mathrm{c}}\left(\mathrm{T}_{1}-\mathrm{M}\right) .
\end{aligned}
$$

Total average cost of the system per unit time is given by

$\mathrm{K}_{1}\left(\mathrm{~T}, \mathrm{~T}_{1}\right)=\frac{1}{T}\left(\right.$ setup cost + Deterioration cost + holding cost + shortages cost $\left.+I C_{1}-I E_{1}\right)$

$\mathrm{K}_{1}\left(\mathrm{~T}, \mathrm{~T}_{1}\right)=\frac{1}{T}\left[\mathrm{~A}+\mathrm{C}\left(\frac{a \theta T_{1}^{3}}{6}+\frac{a \theta^{2} T_{1}^{5}}{40}\right)+\mathrm{h}\left(\frac{a T_{1}^{2}}{2}+\frac{a \theta T_{1}^{4}}{12}+\frac{11 a \theta^{2} T_{1}^{6}}{720}\right)-\frac{a c_{3}}{2}\left(2 \mathrm{TT}_{1}-\mathrm{T}^{2}-\mathrm{T}_{1}^{2}\right)+\right.$ $\left.\frac{a C I_{e} T_{1}^{2}}{2}-\mathrm{CI}_{\mathrm{c}} \mathrm{a}\left(\mathrm{T}_{1}-\mathrm{M}\right)\right]$.

Our objective is to minimize the total cost. The necessary conditions for minimizing the total cost are 
$\frac{\partial K_{1}\left(T, T_{1}\right)}{\partial T_{1}}=0$ and $\frac{\partial K_{1}\left(T, T_{1}\right)}{\partial T}=0$

$\frac{\partial K_{1}\left(T, T_{1}\right)}{\partial T_{1}}=\frac{1}{T}\left[\mathrm{C}\left(\frac{a \theta T_{1}^{2}}{2}+\frac{a \theta^{2} T_{1}^{4}}{8}\right)+\mathrm{h}\left(\mathrm{aT}_{1}+\frac{a \theta T_{1}^{3}}{3}+\frac{11 a \theta^{2} T_{1}^{5}}{120}\right)-\frac{a C_{3}}{2}\left(2 \mathrm{~T}-2 \mathrm{~T}_{1}\right)+\frac{a C I_{e} T_{1}}{2}-\right.$

$\left.\mathrm{CI}_{\mathrm{c}} \mathrm{a}\right]=0$

$\frac{\partial K_{1}\left(T, T_{1}\right)}{\partial T}=\frac{-1}{T^{2}}\left[\mathrm{~A}+\mathrm{C}\left(\frac{a \theta T_{1}^{3}}{6}+\frac{a \theta^{2} T_{1}^{5}}{40}\right) \mathrm{h}\left(\frac{a T_{1}^{2}}{2}+\frac{a \theta T_{1}^{4}}{12}+\frac{11 a \theta^{2} T_{1}^{6}}{720}\right)\right]-\frac{a C_{3}}{2}\left(0-1+\frac{T_{1}^{2}}{T^{2}}\right)-$

$\left(\frac{a C I_{e} T_{1}^{2}}{2 T^{2}}+\frac{C I_{c} a\left(T_{1}-M\right)}{T^{2}}\right\}=0$

Provided they (equations (13) and (14)) satisfy the conditions

$\left(\frac{\partial^{2} K_{1}\left(T, T_{1}\right)}{\partial T^{2}}\right)>0,\left(\frac{\partial^{2} K_{1}\left(T, T_{1}\right)}{\partial T_{1}^{2}}\right)>0 \&\left(\frac{\partial^{2} K_{1}\left(T, T_{1}\right)}{\partial T^{2}}\right)\left(\frac{\partial^{2} K_{1}\left(T, T_{1}\right)}{\partial T_{1}^{2}}\right)-\left(\frac{\partial^{2} K_{1}\left(T, T_{1}\right)}{\partial T \partial T_{1}}\right)>0$

Case II: $M>T_{1}$ (Payment after depletion)

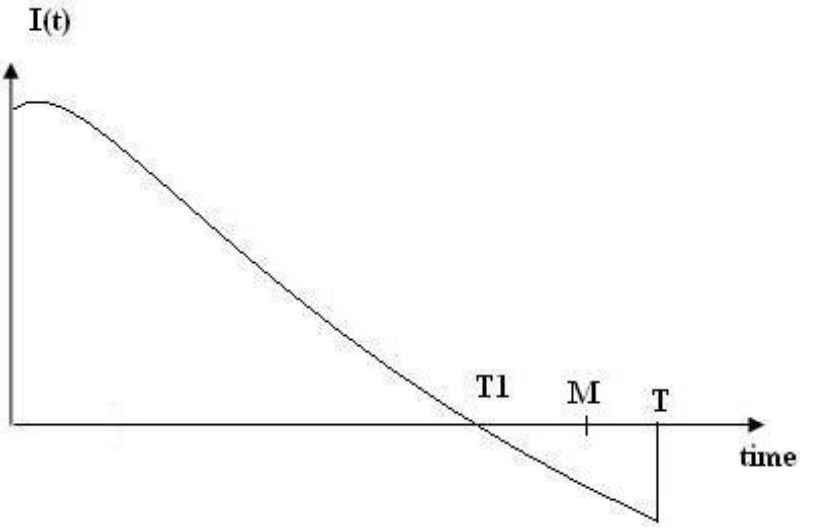

Fig.3

In this case, the interest payable per cycle is zero, $\mathrm{IC}_{2}=0$, when $\mathrm{T}_{1}<\mathrm{M} \leq \mathrm{T}$

$$
\begin{aligned}
\mathrm{IE}_{2}=\mathrm{CI}_{\mathrm{e}}\left[\int_{0}^{T_{1}}\left(T_{1}-t\right)(a) d t+\left(M-T_{1}\right) \int_{0}^{T}(a) d t\right] \\
=\mathrm{CI}_{\mathrm{e}}\left[\mathrm{aMT}_{1}-\frac{a T_{1}^{2}}{2}\right]
\end{aligned}
$$

Total average cost of the system per unit time is given by

$\mathrm{K}_{2}\left(T, T_{1}\right)=\frac{1}{T}\left[\right.$ set up cost + Deterioration cost + holding cost + Shortage cost $\left.-I E_{2}\right]$

$\mathrm{K}_{2}\left(T, T_{1}\right)=\frac{1}{T}\left[\mathrm{~A}+\mathrm{C}\left(\frac{a \theta T_{1}^{3}}{6}+\frac{a \theta^{2} T_{1}^{5}}{40}\right)+\mathrm{h}\left(\frac{a T_{1}^{2}}{2}+\frac{a \theta T_{1}^{4}}{12}+\frac{11 a \theta^{2} T_{1}^{6}}{720}\right)-\frac{a c_{3}}{2}\left(2 \mathrm{TT}_{1}-\mathrm{T}^{2}-\mathrm{T}_{1}^{2}\right)-\mathrm{CI}_{\mathrm{e}}\right.$

$\left.\left(\mathrm{aMT}_{1}-\frac{a T_{1}^{2}}{2}\right)\right]$

Our objective is to minimize the total cost. The necessary conditions for minimizing the total cost are

$$
\begin{aligned}
& \frac{\partial K_{2}\left(T, T_{1}\right)}{\partial T_{1}}=0 \text { and } \frac{\partial K_{2}\left(T, T_{1}\right)}{\partial T}=0 \\
& \frac{\partial K_{2}\left(T, T_{1}\right)}{\partial T_{1}}=\frac{1}{T}\left[\mathrm{C}\left(\frac{a \theta T_{1}^{2}}{2}+\frac{a \theta^{2} T_{1}^{4}}{8}\right)+\mathrm{h}\left(\mathrm{aT}_{1}+\frac{a \theta T_{1}^{3}}{3}+\frac{11 a \theta^{2} T_{1}^{5}}{120}\right)-\frac{a C_{3}}{2}\left(2 \mathrm{~T}-2 \mathrm{~T}_{1}\right)-\mathrm{CI}_{\mathrm{e}}\left(\mathrm{aM}-\mathrm{aT}_{1}\right)\right] \\
& =0
\end{aligned}
$$


$\frac{\partial K_{2}\left(T, T_{1}\right)}{\partial T}=\frac{-1}{T^{2}}\left[\mathrm{~A}+\mathrm{C}\left(\frac{a \theta T_{1}^{3}}{6}+\frac{a \theta^{2} T_{1}^{5}}{40}\right) \mathrm{h}\left(\frac{a T_{1}^{2}}{2}+\frac{a \theta T_{1}^{4}}{12}+\frac{11 a \theta^{2} T_{1}^{6}}{720}\right)\right]-\frac{a C_{3}}{2}\left(-1+\frac{T_{1}^{2}}{T^{2}}\right)+$

$\left(\frac{C I_{e}\left(a M T_{1}\right)}{T^{2}}-\frac{\left.C I_{e} a T_{1}^{2}\right)}{2 T^{2}}\right)=0$

Provided they (equations (17) \& (18)) satisfy the conditions

$\left(\frac{\partial^{2} K_{2}\left(T, T_{1}\right)}{\partial T_{1}^{2}}\right)>0,\left(\frac{\partial^{2} K_{2}\left(T, T_{1}\right)}{\partial T^{2}}\right)>0$ and $\left(\frac{\partial^{2} K_{2}\left(T, T_{1}\right)}{\partial T^{2}}\right)\left(\frac{\partial^{2} K_{2}\left(T, T_{1}\right)}{\partial T_{1}^{2}}\right)-\left(\frac{\partial^{2} K_{2}\left(T, T_{1}\right)}{\partial T_{1} \partial T}\right)>0$.

\section{Case I: $\mathbf{M} \leq \mathbf{T}_{\mathbf{1}}$}

\section{NUMERICAL EXAMPLES}

Let us take $A=1000, a=300, h=5, \theta=0.6, C_{3}=0.8, I_{e}=0.09, M=0.08, I_{c}=0.12, C=20$ in the respective proper units. Then we get $T_{1}=0.4801$ is greater than $M=0.08$. Also $T=2.9532$,

$T C=593.5394$ and $Q=247.4187$

\section{Case II: $\mathbf{M}>\mathbf{T}_{1}$}

Let us take $A=1000, a=300, h=5, \theta=0.6, C_{3}=0.8, I_{e}=0.09, M=0.35, C=20$ in the respective proper units. Then we get $T_{1}=0.3143$ is less than $M=0.35$. Also $T=2.9899, T C=642.1459$ and $Q=$ 95.2297

\section{SENSITIVITY ANALYSIS}

Sensitivity analysis has been carried out by keeping one variable changing while all other variables as constant.

Table 1

\begin{tabular}{|c|c|c|c|c|}
\hline $\mathrm{h}$ & $\mathrm{T}_{1}$ & $\mathrm{~T}$ & $\mathrm{TC}$ & $\mathrm{Q}$ \\
\hline 6 & 0.4456 & 2.9588 & 603.1786 & 136.3818 \\
\hline 7 & 0.4148 & 2.9627 & 611.5131 & 126.6143 \\
\hline 8 & 0.3873 & 2.9655 & 618.7733 & 117.9564 \\
\hline 9 & 0.3627 & 2.9675 & 625.1404 & 110.2584 \\
\hline & & & & \\
\hline$\theta$ & $\mathrm{T}_{1}$ & $\mathrm{~T}$ & $\mathrm{TC}$ & $\mathrm{Q}$ \\
\hline 0.7 & 0.4615 & 2.9475 & 596.6431 & 141.9671 \\
\hline 0.8 & 0.4452 & 2.9430 & 599.4622 & 137.1735 \\
\hline 0.9 & 0.4308 & 2.9393 & 602.0433 & 132.9280 \\
\hline 1.0 & 0.4178 & 2.9362 & 604.4248 & 129.0820 \\
\hline $\mathrm{a}$ & $\mathrm{T}_{1}$ & $\mathrm{~T}$ & TC & $\mathrm{Q}$ \\
\hline 350 & 0.4667 & 2.7278 & 633.0839 & 166.9725 \\
\hline 400 & 0.4557 & 2.5453 & 668.6476 & 186.1360 \\
\hline 450 & 0.4464 & 2.3935 & 700.9201 & 204.9548 \\
\hline 500 & 0.4385 & 2.2645 & 730.4170 & 223.5387 \\
\hline $\mathrm{C}_{3}$ & $\mathrm{~T}_{1}$ & $\mathrm{~T}$ & TC & $\mathrm{Q}$ \\
\hline 0.7 & 0.4715 & 3.1414 & 560.6751 & 144.6575 \\
\hline 0.6 & 0.4619 & 3.3757 & 524.4924 & 141.5832 \\
\hline 0.5 & 0.4510 & 3.6785 & 484.1174 & 138.1024 \\
\hline 0.4 & 0.4385 & 4.0903 & 438.2180 & 134.1232 \\
\hline
\end{tabular}

Table - 2

\begin{tabular}{|c|c|c|c|c|}
\hline $\mathrm{h}$ & $\mathrm{T}_{1}$ & $\mathrm{~T}$ & $\mathrm{TC}$ & $\mathrm{Q}$ \\
\hline 6 & 0.2886 & 2.9833 & 646.7447 & 87.3065 \\
\hline 7 & 0.2662 & 2.9772 & 650.6418 & 80.4295 \\
\hline 8 & 0.2467 & 2.9716 & 653.9757 & 74.4629 \\
\hline 9 & 0.2295 & 2.9664 & 656.8530 & 69.2144 \\
\hline & & & & $\mathrm{TC}$ \\
\hline$\theta$ & $\mathrm{T}_{1}$ & $\mathrm{~T}$ & 643.1991 & 92.6267 \\
\hline 0.7 & 0.3054 & 2.9854 & 644.1701 & 90.2523 \\
\hline 0.8 & 0.2973 & 2.9814 & 645.0703 & 88.1100 \\
\hline 0.9 & 0.2900 & 2.9778 & 645.9087 & 86.1093 \\
\hline
\end{tabular}




\begin{tabular}{|c|c|c|c|c|}
\hline & & & & \\
\hline $\mathrm{a}$ & $\mathrm{T}_{1}$ & $\mathrm{~T}$ & $\mathrm{TC}$ & $\mathrm{Q}$ \\
\hline 350 & 0.2991 & 2.7680 & 691.2791 & 105.6291 \\
\hline 400 & 0.2866 & 2.5888 & 736.6964 & 115.5886 \\
\hline 450 & 0.2761 & 2.4402 & 779.0716 & 125.1986 \\
\hline 500 & 0.2671 & 2.3143 & 818.8957 & $\mathrm{Q}$ \\
\hline & & & & $\mathrm{TC}$ \\
\hline $\mathrm{C}_{3}$ & $\mathrm{~T}_{1}$ & $\mathrm{~T}$ & 604.6992 & 81.7106 \\
\hline 0.7 & 0.3029 & 3.1824 & 563.7346 & 83.3737 \\
\hline 0.6 & 0.2901 & 3.4220 & 518.3469 & 78.2856 \\
\hline 0.5 & 0.2758 & 3.7314 & 467.1546 & \\
\hline 0.4 & 0.2592 & 4.1521 & & \\
\hline
\end{tabular}

\section{Conclusion}

This model developed in this paper assumes that demand of the product is constant and deteriorating rate is time proportional. The benefit of the trade credit for the retailer is illustrated with numerical example and sensitivity analysis. It can be noted down that increase of holding cost, deterioration rate and demand give increase in total cost. Similarly decrease in shortage cost decreases the total cost for case (I). For Case (II) also increase in the parameters increases the total cost and decreases causes decrease in the total cost.

\section{REFERENCES}

[1] Ajanta Roy (2008) "An inventory model for deteriorating items with price dependent demand and time-varying holding cost." AMOAdvanced Modeling and Optimization, volume 10, November 1

[2] Chaitanya Kumar Tripathy and Umakanta Mishra, "Ordering Policy for Weibull Deteriorating Items for Quadratic Demand with Permissible Delay in Payments", Applied Mathematical Sciences, Vol.4, 2010, no.44, 2181-2191.

[3] Hardik Soni, Nita H.Shan and Chandra K.Jaggi, "Inventory models and trade credit: a review", Control and Cybernetics, Vol39, (2010) No.3.

[4] Hesam Saiedy, Mohammed Bamani Moghadam, "Inventory Model with Composed Shortage and permissible delay in payment linked to order quantity", J. Ind. Engg. Int.7(15) 1-7, Fall 2011, ISSN: 1735-5702

[5] Jamal AMM, BR Sarker and S Wang, "An ordering policy for deteriorating items with allowable shortage and permissible delay in payment", Journal of the Operational Research Society (1997), 48, 826-833.

[6] Khanr.S, K.S.Chaudhuri, "A Production - Inventory Model for a Deteriorating Item with Shortage and Time- Dependent Demand", Yugoslav Journal of Operations Research 21 (2011), Number 1, 29-45.

[7] Kumar Arya.R, and S.Kumar Shakya, "An Inventory Model for Deteriorating Items with Time Dependent Demand and Partial Backlogging”, Int.J.of Appl.Math and Mech. 8 (8): 55-71, 2012.

[8] Kun -Shan Wu “An Ordering Policy for Items with Weibull distribution Deterioration under permissible Delay in Payments." Tamsui Oxford Journal of Mathematical Science 14 (1998) 39-54.

[9] Manisha Pal and Sanjoy Kumar Ghosh, "An Inventory Model with Shortage and Quantity Dependent Permissible Delay in payment",

[10] Manoj Kumar Meher, Gobinda Chandra Panda, Sudhir Kumar Sahu," An Inventory Model with Weibull Deterioration Rate under the Delay in Payment in Demand Decling Market" Applied Mathematical Sciences,Vol.6, 2012, no.23, 1121 -1133.

[11] Maryam Ghoreishi, Alireza Arshsadi Khamseh, Abolfazl Mirzazadeh, "Joint Optimal Pricing and Inventory Control for Deteriorating Items under Inflation and Customer Returns",

[12] Mukesh Kumar, Anand Chauhan, Rajat Kumar, "A Deterministic Inventory Model for Deteriorating Items with Price Dependent Demand and Time Varying Holding Cost under Trade Credit”, International Journal of Soft Computing Engineering (IJSCE), ISSN: 2231-2307, Volume - 2, Issue-1, March 2012.

[13] Nita H.Shah, Poonam Pandey, " Optimal Ordering Policy for the Time Dependent Deterioration with Associated Salvage Value when Delay In payments is Permissible", Revista Investigacion Operacional, Vol.2, No.2, 117-129, 2008

[14] Ouyang L-Y, C-T Chang and J-T Teng, "An EOQ model for deteriorating items under trade credits", Journal of the Operational Research Society (2205) 56, $719-726$.

[15] Ravi Gor, Nita H Shah "An EOQ Model for Deteriorating Items with price Dependent Demand and Permissible Delay in Payments under Inflation”, Opsearch, Vol.43, No.4, 2006.

[16] Sanjay Jain, Mukesh Kumar and Priya Advani, “An Inventory Model with Inventory Level-Dependent Demand Rate, Deterioration, Partial Backlogging and Decrease in Demand", International Journal of Operations Research , Vol.5, No.3, 154-159 (2008)

[17] Shital S.Patel and R.D.Patel, "EOQ Model for Weibull Deteriorating Items with Linear Demand under Permissible Delay in Payments", International Journal of Computational Science and Mathematics, ISSN 0974-3189, Volume 4, Number 3 (@012), PP. 175-185.

[18] Srichandan Mishra, L.K.Raju, U.K.Misra and G.Misra, "A Study of EOQ Model with Power Demand of Deteriorating Items under the Influence of Inflation”, Gen. Math. Notes. Vol.10, No.1, May 2012, pp: 41 -50, ISSN 2219-7184.

[19] Trailokyanath Singh, Hadibandhu Pattanayak "An EOQ Model for A Deteriorating Item with Time Dependent Exponentially Declining Demand Under Permissible Delay In payment” IOSR Journal of Mathematics (IOSRJM) ISSN: 2278 -5728 Volume 2, Issue 2 (July -Aug 2012), pp 30 -37

[20] Tripathi R.P., " Optimal pricing and ordering policy for inflation dependent demand rate under permissible delay in payments" .International Journal of Business, Management and Social sciences, vol. 2,No.4, 2011,pp 35-43.

[21] Tripathi R.P., S.S.Misra, H.S.Shukla "A Cash flow oriented EOQ model under permissible delay in payments" International Journal of Engineering, Science and Technology Vol.2, No.11, 2010, pp.123-131.

[22] Tripathy P.K, S.Pradhan "An Integrated Partial Backlogging Inventory Model having Weibull Demand and Variable Deterioration rate with the Effect of Trade Credit." International Journal of Scientific \& Engineering Research Volume 2, Issue 4, April-2011, ISSN $2229-5518$ 